\title{
AGV Trajectory Control Based on Laser Sensor Navigation
}

\author{
Thanh Luan Bui ${ }^{1}$, Phuc Thinh Doan ${ }^{1}$, Soon Sil Park ${ }^{2}$, Hak Kyeong Kim ${ }^{1}$ and ${ }^{*}$ Sang Bong Kim ${ }^{1}$ \\ ${ }^{1}$ Department of Mechanical and Automotive Eng., Pukyong National University, Busan 608-739, Korrea \\ ${ }^{2}$ Ranault Samsung Motors, Busan, Korea. \\ Corresponding author, Tel: +82-51-629-6158, \\ E-mail: kimsb@pknu.ac.kr
}

\begin{abstract}
Autonomous Guided Vehicle Systems (AGVs) are used to transport goods and products in manufacturing fields where navigation can be done in a structured environment. In order to track the given trajectory, a tracking control based on Lyapunov stability theory is introduced. The use of the nonlinear Lyapunov technique provides robustness for load disturbance and sensor noise. To apply Lyapunov's theorem, the kinematic model of AGV is given. To recognize its position in indoor environment, in this paper, a laser sensor device NAV200 is used to detect the AGV position in realtime. For simulation and experiment, software and hardware are described. The AGV consists of 4 wheels with two passive wheels and two driving wheels. A controller is developed based on industrial computer. The effectiveness of the proposed controller is proved by simulation and experimental results.
\end{abstract}

\section{INTRODUCTION}

Automated Guided Vehicle (AGV) is a transportation vehicle automatically traveling on a predefined route. AGV is most often used to deliver materials around a manufacturing facility or a warehouse. With lots of advantages such as reduction in the number of worker, improvement of productivity and quality, improvement of work environment and safety, less damage on transporting goods, real-time control of material flow and improved management on product, AGV has attracted the attention to many researchers as well as manufacturers [1, 3, 5, 6]. O.H. Dagci et. al. [2] introduced a controller using sliding mode control theory for mobile robot. Nazari et. al. [4] introduced a control method based on Fuzzy logic for path following of skid steer mobile robots. Malik Arjuna et al. [7] and Dung et. al. [8] proposed an adaptive sliding mode control technique for two-wheeled welding for mobile robot. However, their systems have not yet applied to heavy industrial vehicles. A trajectory tracking controller is needed for heavy industrial vehicle.

This paper is about control of an industrial AGV for tracking a reference trajectory using laser sensor NAV200. First, the AGV is moved manually to track the reference trajectory. At that time, the coordinates of the AGV along the reference trajectory obtained from laser sensor is stored into memories. This data will be used to help AGV move repeatedly their trajectory in the future. A trajectory tracking control method is also proposed for $\mathrm{AGV}$ based on Lyapunov technique. The followings are done for this task. The first one is system description, the second one is AGV's modeling and the third is to design a controller for trajectory tracking of AGV. Finally, simulation and experimental results on the AGV with 4 wheels are carried out to evaluate the effectiveness of the proposed controller.

\section{SYSTEM DESCRIPTION AND MODELING}

2.1 System description

The AGV has 4 wheels, two passive wheels and two driving wheels. The driving wheel is directly driven by two Brushless DC motors. The laser sensor NAV200 is set on the top of the AGV. Fig.1 shows the controller system that is developed based on the integration of a computer industry and PCI card. Computer industry and laser sensor communicate each other through RS232 protocol. A control panel and monitor touch screen is used. AGV can operate in both manual and automatic mode.

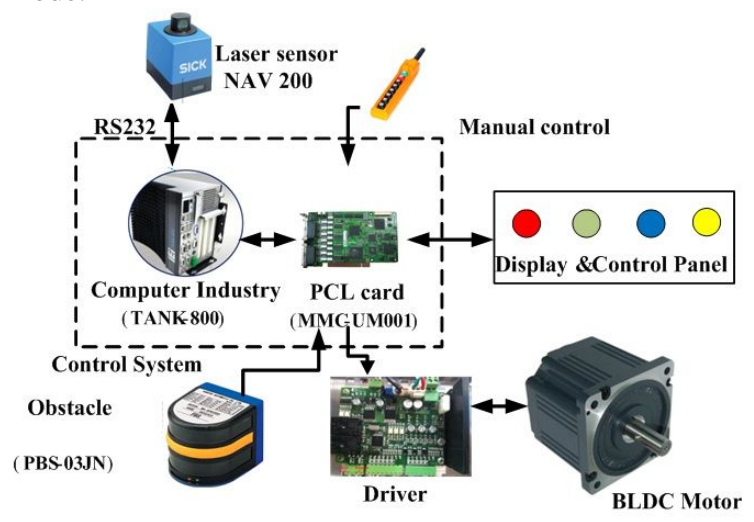

Fig.1 Schematic of the control system for the AGV

A control system is developed based on computer industry TANK-800 which is operated with the clock $1.8 \mathrm{GHz}$ dual-core processor and PCI card MMC-BDPV42PNX 4-axis controller. The NAV200 system is a laser-based positioning that returns an absolute position of the scanner with respect to a user-defined local coordinate frame. On average, this system can provide up to millimeter accuracy with an update rate up to $8 \mathrm{~Hz}$. The prototype of the experimental AGV is shown in Fig.2.

System modeling

The AGV's architecture together with its symbols is shown in Fig.3 where it is supposed that its point of geometric centre C and the centre of gravity coincide. 


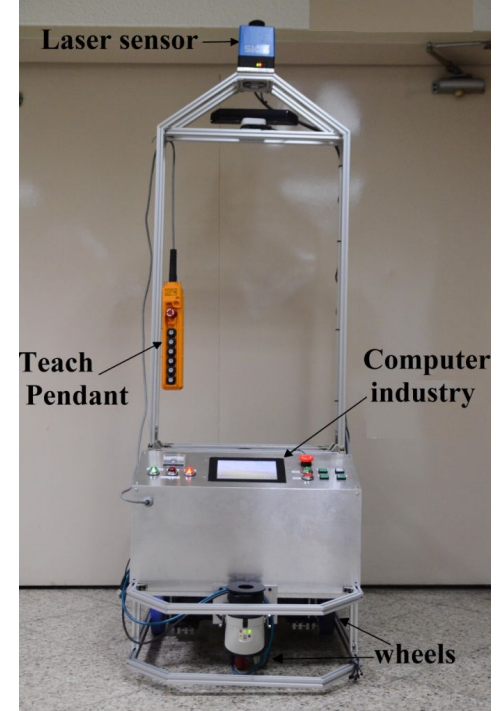

Fig.2 Prototype of the experimental AGV

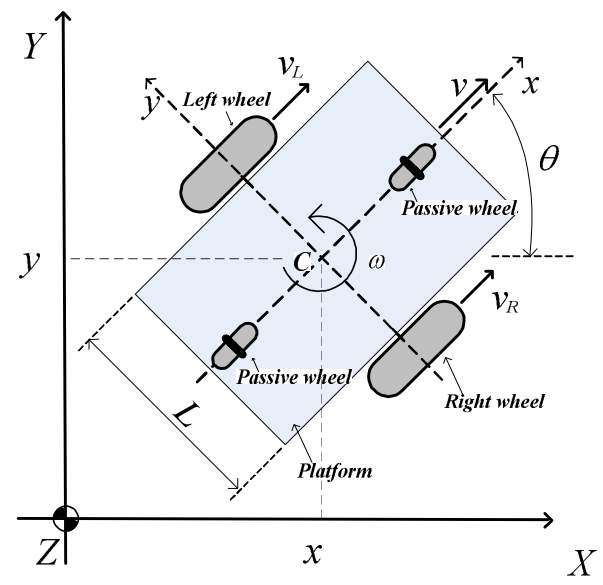

Fig.3 AGV architecture and its symbol

The kinematic equations of the AGV are as follows:

$$
\dot{\mathbf{q}}=\left[\begin{array}{c}
\dot{x} \\
\dot{y} \\
\dot{\theta}
\end{array}\right]=\left[\begin{array}{cc}
\cos \theta & 0 \\
\sin \theta & 0 \\
0 & 1
\end{array}\right]\left[\begin{array}{l}
v \\
\omega
\end{array}\right]
$$

where $\dot{\mathbf{q}}=[x, y, \theta]^{T}$ is a position vector of AGV, $v$ and $\omega$ are linear and angular velocities of the platform in Fig.3. The velocities of the right and the left wheel of the AGV are

$$
v_{R}=v+\frac{\omega L}{2} \quad v_{L}=v-\frac{\omega L}{2}
$$

where $L$ is the AGV inter-wheel distance.

For a given reference trajectory $\left(x_{r}(t), y_{r}(t)\right)$, the AGV reference linear velocity $\mathrm{vr}$ and reference angular velocity $\omega \mathrm{r}$ are calculated from the reference trajectory.

The reference linear velocity can be calculated as $v_{r}(t)= \pm \sqrt{\dot{x}_{r}^{2}(t)+\dot{y}_{r}^{2}(t)}$

where the sign depends on drive direction (+ for forward and for reverse). The reference angle of each point on the trajectory is defined as

$\theta_{r}(t)=\arctan 2\left(\dot{y}_{r}(t), \dot{x}_{r}(t)\right)+k \pi$

where $k=0,1$ defines the desired direction ( 0 for forward and 1 for reverse) and function arctan 2 is the inverse tangent function which returns the correct angles in all situations.

By calculating the time derivative of Eq. (4), the AGV angular velocity is obtained as follows:

$\omega_{r}(t)=\frac{\dot{x}_{r}(t) \ddot{y}_{r}(t)-\ddot{x}_{r}(t) \dot{y}_{r}(t)}{\dot{x}_{r}^{2}(t)+\dot{y}_{r}^{2}(t)}=v_{r}(t) k(t)$

where $k(t)$ is trajectory curvature. Using Eqs. $(1 \div 5)$ and the defined reference trajectory $\mathrm{qr}(\mathrm{t})=\left[x_{r}(t), y_{r}(t), \theta_{r}(t)\right] \mathrm{T}$, $v_{r}(t)$ and $\omega_{r}(t)$ are calculated. The necessary condition in the path design procedure is to take twice differentiable path and nonzero $\mathrm{AGV}$ reference velocity $v_{r}(t) \neq 0$. If for some time ${ }^{t}$, AGV reference velocity $v_{r}(t)=0$, the AGV rotates at a fixed point with the reference $\mathrm{AGV}$ angular velocity $\omega_{r}(t)$.

\section{CONTROLLER DEGIGN}

When the AGV is controlled to drive on a reference trajectory, it usually has some tracking error. In Fig.4, the tracking error vector $\mathbf{e}=\left[e_{1}, e_{2}, e_{3}\right]^{T}$ is defined as the difference between the centre point of $\mathrm{AGV}$ and the reference point.

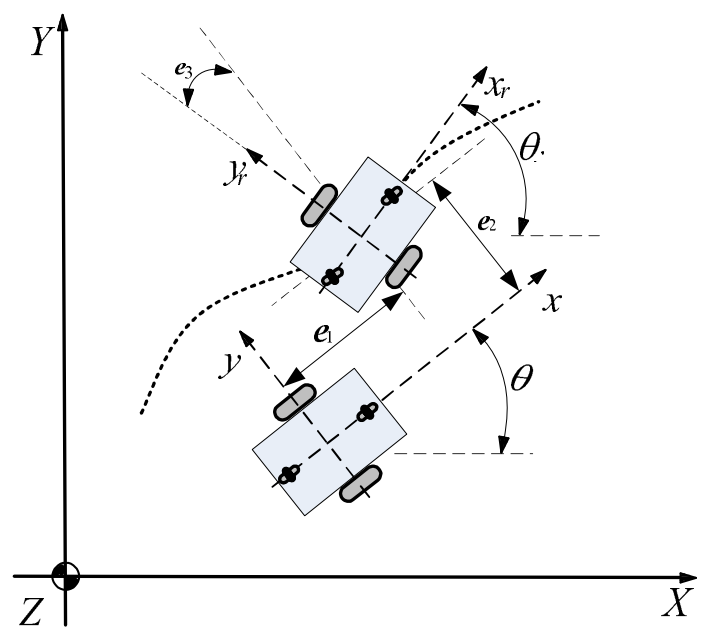

Fig.4 Configuration for kinematic model of the AGV 
$\mathbf{e}=\left[\begin{array}{l}e_{1} \\ e_{2} \\ e_{3}\end{array}\right]=\left[\begin{array}{ccc}\cos \theta & \sin \theta & 0 \\ -\sin \theta & \cos \theta & 0 \\ 0 & 0 & 1\end{array}\right]\left[\begin{array}{l}x_{r}-x \\ y_{r}-y \\ \theta_{r}-\theta\end{array}\right]$

A control algorithm should be designed to force the AGV to track the reference trajectory precisely.

Using the robot kinematics Eq. (1) and the tracking error vector Eq. (6), the following error dynamics is obtained

$\dot{\mathbf{e}}=\left[\begin{array}{l}\dot{e}_{1} \\ \dot{e}_{2} \\ \dot{e}_{3}\end{array}\right]=\left[\begin{array}{cc}\cos e_{3} & 0 \\ \sin e_{3} & 0 \\ 0 & 1\end{array}\right]\left[\begin{array}{l}u_{r 1} \\ u_{r 2}\end{array}\right]+\left[\begin{array}{cc}-1 & e_{2} \\ 0 & -e_{1} \\ 0 & -1\end{array}\right]\left[\begin{array}{l}u_{1} \\ u_{2}\end{array}\right]$

where ur1 = vr is feedforward linear velocity Eq. (3) and ur2 = $\omega r$ is feedforward angular velocity Eq. (5). An AGV input vector can be expressed in the following form

$\mathbf{u}=\left[\begin{array}{c}v \\ w\end{array}\right]=\left[\begin{array}{c}u_{r 1} \cos e_{3} \\ u_{r 2}\end{array}\right]-\left[\begin{array}{l}v_{1} \\ v_{2}\end{array}\right]$

where the first parts on the right side of Eq. (8) are the feed forward inputs, while v1 and $\mathrm{v} 2$ are new inputs obtained from the closed loop. Substituting Eq. (8) in to Eq. (7) yields:

$$
\begin{aligned}
& {\left[\begin{array}{c}
\dot{e}_{1} \\
\dot{e}_{2} \\
\dot{e}_{3}
\end{array}\right]=\left[\begin{array}{ccc}
0 & u_{2} & 0 \\
-u_{2} & 0 & 0 \\
0 & 0 & 0
\end{array}\right]\left[\begin{array}{l}
e_{1} \\
e_{2} \\
e_{3}
\end{array}\right]} \\
& +\left[\begin{array}{c}
0 \\
\sin e_{3} \\
0
\end{array}\right] u_{r 1}+\left[\begin{array}{ll}
1 & 0 \\
0 & 0 \\
0 & 1
\end{array}\right]\left[\begin{array}{l}
v_{1} \\
v_{2}
\end{array}\right]
\end{aligned}
$$

Linearizing Eq. (9) around the operating point $\left(e_{1}=e_{2}=e_{3}=0, v_{1}=v_{2}=0\right)$ linear model:

$$
\begin{aligned}
& \dot{\mathbf{e}}=\left[\begin{array}{c}
\dot{e}_{1} \\
\dot{e}_{2} \\
\dot{e}_{3}
\end{array}\right]=\left[\begin{array}{ccc}
0 & u_{r 2} & 0 \\
-u_{r 2} & 0 & u_{r 1} \\
0 & 0 & 0
\end{array}\right]\left[\begin{array}{l}
e_{1} \\
e_{2} \\
e_{3}
\end{array}\right] \\
&+\left[\begin{array}{ll}
1 & 0 \\
0 & 0 \\
0 & 1
\end{array}\right]\left[\begin{array}{c}
v_{1} \\
v_{2}
\end{array}\right]=\overline{\mathbf{A}} \mathbf{e}+\overline{\mathbf{B}} \mathbf{v}
\end{aligned}
$$

where $\mathbf{v}=\left[\begin{array}{ll}v_{1} & v_{2}\end{array}\right]^{T}$ is a new control input vector.

A new controller for the closed loop is designed as

$$
\mathbf{v}=\mathbf{K} \mathbf{e}
$$

where gain matrix $\mathbf{K}$ has dimension $2 \times 3$.

The control is explained in the block diagram of the proposed
AGV control system as shown in Fig.5. To reduce error in the driving direction $\mathrm{e} 1$, the reference linear velocity of the AGV should be changed correspondingly. Similarly, the orientation error e3 can be efficiently manipulated by AGV angular velocity. Finally, the error orthogonal to the driving direction could be reduced by changing the angular velocity. At the same time the AGV drive direction (forward or backward) should be considered. From the above conclusion, the control law of Eq. (11) can be given as

$$
\mathbf{v}=\left[\begin{array}{l}
v_{1} \\
v_{2}
\end{array}\right]=\left[\begin{array}{ccc}
-k_{1} & 0 & 0 \\
0 & -\operatorname{sgn}\left(u_{r 1}\right)\left|u_{r 1}\right| k_{2} & -k_{3}
\end{array}\right]\left[\begin{array}{l}
e_{1} \\
e_{2} \\
e_{3}
\end{array}\right]
$$$$
=\mathbf{K} \mathbf{e}
$$

where $\mathbf{u}_{r}=\left[\begin{array}{ll}u_{r 1} & u_{r 2}\end{array}\right]^{T}=\left[\begin{array}{ll}v_{r} & \omega_{r}\end{array}\right]^{T}$ is a feed-forward velocity vector.

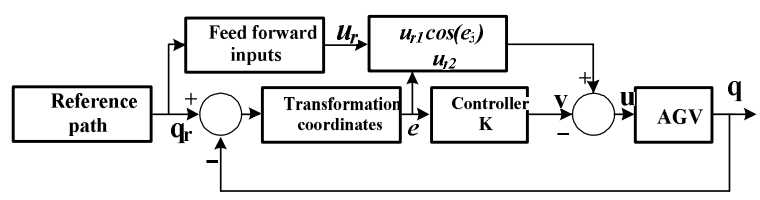

Fig.5 Block diagram of the proposed AGV control system

\section{STABILITY ANALYSIS}

For the presented controller Eqs. $(8 \div 12)$, the stability in the sense of Lyapunov is shown in this section. Lyapunov function $V(\mathbf{e})$ should be positive definite and continuous with continuous derivatives on an tracking error vector $\mathbf{e}$ In the equilibrium point $\mathbf{e}=0, V(\mathbf{e})=0$

The origin point $\mathbf{e}=0$ in error dynamics is stable at equilibrium point if there exist $V(\mathbf{e})$ that $\dot{V}(\mathbf{e})$ is negative semidefinite over all the trajectory. If $\dot{V}(\mathbf{e})$ is negative definite, the equilibrium point is asymptotically stable.

If we choose $k_{2}>0$, the new controller of Eq. (12) becomes

$v_{1}=-k_{1} e_{1}$

$v_{2}=-k_{2} u_{r 1} e_{2}-k_{3} e_{3}$

Lyapunov function is defined as

$V(\mathbf{e})=\frac{k_{2}}{2}\left(e_{1}^{2}+e_{2}^{2}\right)+\frac{e_{3}^{2}}{2}$

and its derivative as

$V(\mathbf{e})=\frac{k_{2}}{2}\left(e_{1}^{2}+e_{2}^{2}\right)+\frac{e_{3}^{2}}{2}$

From Eq. (10) and the new controller Eq. (13), the following relation is obtained 
$\dot{V}(\mathbf{e})=-k_{1} k_{2} e_{1}^{2}-k_{3} e_{3}^{2} \leq 0$

where controller gains are $k_{1}>0$ and $k_{3}>0$ When the $\mathrm{AGV}$ is in parallel with the reference line trajectory, $e_{1}=e_{3}=0$ and $e_{2} \neq 0$. In this situation the linear controller Eq. (13) can't force $e_{2}$ to zero.

\section{SIMULATION AND EXPERIMENTAL RESULTS}

Simulation and experiment are done on AGV system with parameter $L=0.6 \mathrm{~m}$. Initial conditions are $\mathrm{x} 0=9 \mathrm{~m}, \mathrm{y} 0=9 \mathrm{~m}$, $\theta 0=0, \omega 0=0, \mathrm{vr}=40 \mathrm{~mm} / \mathrm{s}, \omega \mathrm{r} 0=0, \mathrm{k} 1=\mathrm{k} 3=2.4$ and $\mathrm{k} 2=39.2$.

Fig.6 shows the reference trajectory for simulation and experiment. Fig.7 shows the position error e1 that is bounded around $\pm 10 \mathrm{~mm}$ in experiment and $\pm 2.5 \mathrm{~mm}$ in simulation. Fig. 8 shows the position error e2 that is bounded around $\pm 10 \mathrm{~mm}$ in experiment and $\pm 3 \mathrm{~mm}$ in simulation. Fig.9 shows the position error e3 that is bounded around \pm 0.35 rad in experiment and $\pm 0.1 \mathrm{rad}$ in simulation. Fig. 10 shows velocities of left wheel and right wheel.

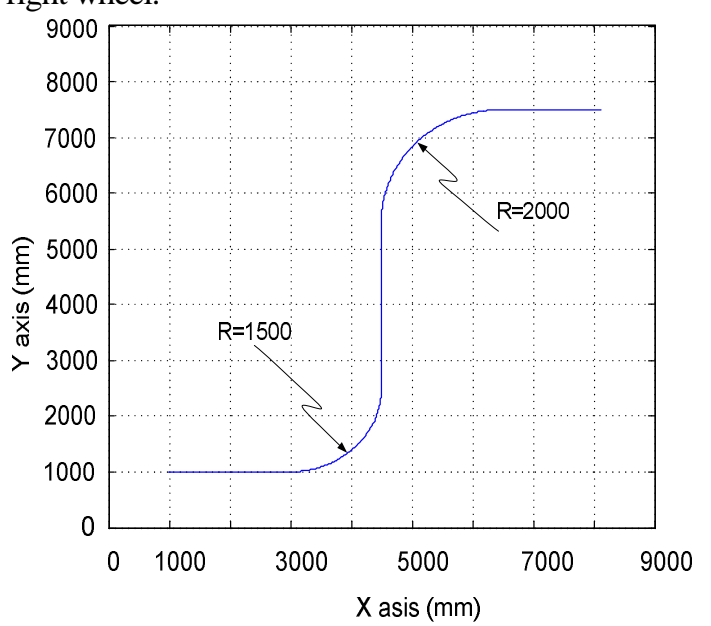

Fig.6 Reference trajectory

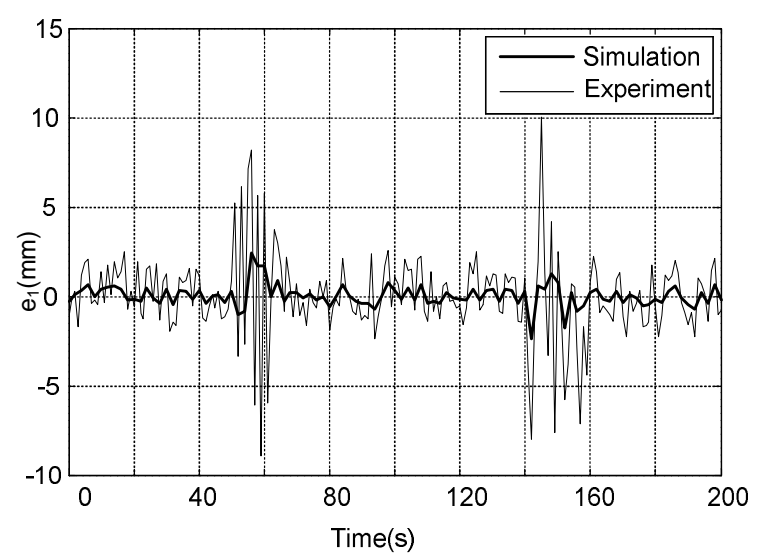

Fig.7 Position error e1

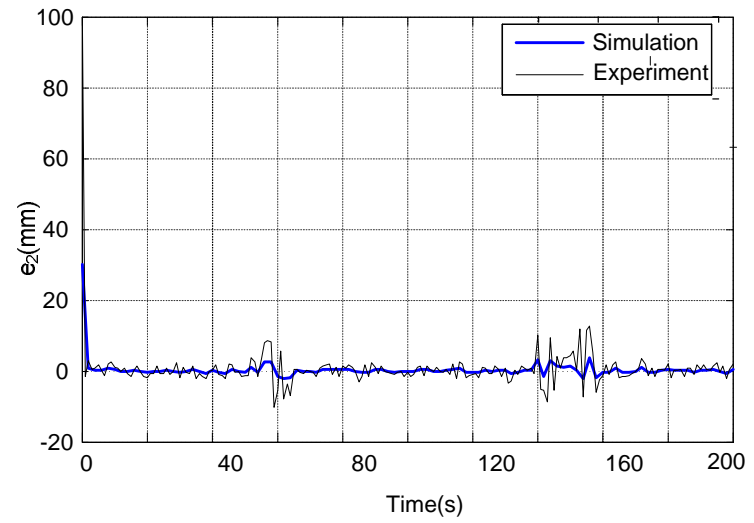

Fig.8 Position error e2

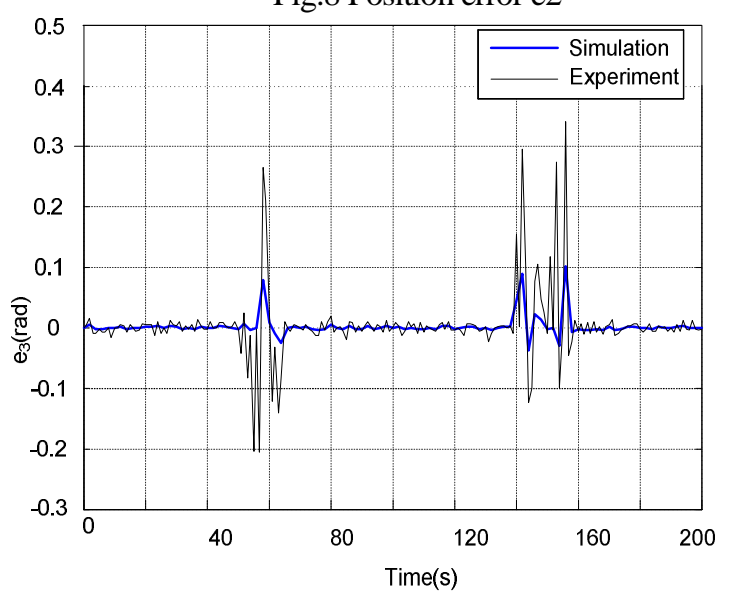

Fig.9 Position error e3

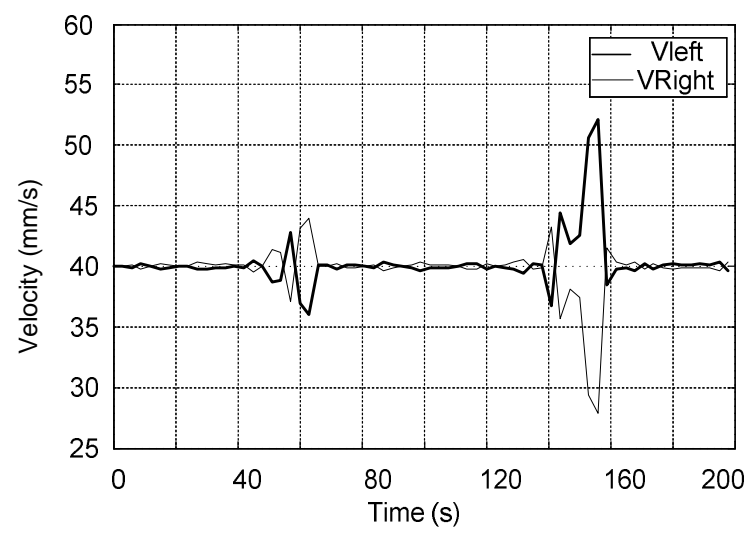

Fig.10 Velocities of left wheel and right wheel

\section{CONCLUSIONS}

In this paper, an industrial $\mathrm{AGV}$ based on laser sensor is developed. First, the system modeling was given. After that, a kinematic nonlinear controller based on Lyapunov stability theory for trajectory tracking was proposed. The simulation and experimental results proved that the proposed controller can obtain a good performance. The AGV tracks the reference trajectory smoothly with small errors.

\section{ACKNOWLEDGEMENT}

This research was supported by a grant from Construction Technology Innovation Program (CTIP) funded by Ministry of Land, Transportation and Maritime Affairs (MLTM) of Korean government. 


\section{REFERENCES}

[1] G. Campion, G. Bastin and B.D. Andreá-Novel, Structural Properties and Classification of Kinematic and Dynamic Models of Wheeled Mobile Robots, IEEE Transactions on Robotics and Automation, Vol. 12 (1996), pp. 47-62.

[2] O.H. Dagci, U.Y. Ograg and U. Ozgiiner, Path Following Controller Design Using Sliding Mode Control Theory, Proceedings of the American Control Conference Denver, Colorado (2003), pp. 903-908.

[3] M. Eghtesad and D.S. Necsulescu, Experimental Study of the Dynamic based Feedback Linearization of an Autonomous Wheeled Ground Vehicle, Elsevier Journal of Robotics and Autonomous Systems, Vol. 47 (2004), pp. 47-63.

[4] V. Nazari and M. Naraghi, Sliding Mode Fuzzy Control of a Skid Steer Mobile Robot for Path Following, Hanoi, Vietnam December (2008), pp. 549-554.

[5] H. Maaref and C. Barret, Sensor-Based Fuzzy Navigation of an
Autonomous Mobile Robot in an Indoor Environment, Contr. Eng. Practice, Vol. 88, July (2000), pp. 757-76.

[6] T.L. Chung, T.H. Bui, T.T. Nguyen and S.B. Kim, Sliding Mode Control of Two Wheeled Welding Mobile Robot for Tracking Smooth Curved Welding Path, International Journal of KSME, Vol. 18, No. 7, April (2007), pp. 1094-1106.

[7] Mallikarjuna Rao G, Vijaya Kumari G, Babu G R, Rajesh Viswanadula. Fast Local Binary Patterns for Efficient Face Recognition, International Journal of Science and Engineering (IJSE), Vol. 2(2)2011, pp.22-26

[8] N.M. Dung, V.H. Duy, N.T. Phuong, S.B. Kim and M.S. Oh, Twowheeled Welding Mobile Robot for Tracking a Smooth Curved Welding Path Using Adaptive Sliding Mode Control Technique, Interna-tional Journal of Control, Automation and Systems (IJCAS), Vol. 5, No. 3, June (2007), pp. 283-94. 